\title{
Correlative study between the JAK2V617F mutation and thrombosis in patients with myeloproliferative neoplasm
}

\author{
Z.C. Li ${ }^{1,2 *}$, H.J. Fu ${ }^{3 *}$, Z.M. Wang ${ }^{2}$, S. Yang ${ }^{2}$ and H.Z. Xu ${ }^{1}$ \\ ${ }^{1}$ Department of Hematology, Provincial Hospital, Shandong University, \\ Jinan, China \\ ${ }^{2}$ Binzhou People's Hospital, Binzhou, China \\ ${ }^{3}$ Binzhou Medical University Hospital, Binzhou, China \\ *These authors contributed equally to this study. \\ Corresponding author: H.Z. Xu \\ E-mail: xuhongzhi1965@sina.com
}

Genet. Mol. Res. 15 (3): gmr.15038423

Received January 12, 2016

Accepted March 28, 2016

Published August 29, 2016

DOI http://dx.doi.org/10.4238/gmr.15038423

Copyright (C) 2016 The Authors. This is an open-access article distributed under the terms of the Creative Commons Attribution ShareAlike (CC BY-SA) 4.0 License

\begin{abstract}
In this study, we investigated the correlation between the JAK2V617F mutation and thrombosis in patients with myeloproliferative neoplasm (MPN) using real-time fluorescence quantitative PCR. The incidence of thrombus was monitored and blood and coagulation were routinely assayed in patients with MPN. The JAK2V617F mutation was found in 8/68 individuals in the control group (11.8\%); it was expressed in 44/68 patients with MPN (64.7\%), suggesting that the rate of this mutation was significantly higher in patients with MPN than that in the control group. Twenty-six MPN patients (38.2\%) showed symptoms of thrombosis; MPN patients with thrombosis showed a significantly higher rate of the JAK2V617F mutation, were of a greater age, and had higher blood pressure than MPN patients without thrombosis. In
\end{abstract}


addition, the white blood cells (WBC) $(21.98 \pm 1.95)$ and platelets (364.68 \pm 97.72$)$ were significantly higher in patients, expressing the mutated gene, with polycythemia vera than in the patients without the mutation. The WBC $(32.89 \pm 4.25)$ and hemoglobin $(161.92 \pm 16.19)$ were significantly increased in the essential thrombocythemia patients with gene mutation compared with the patients without mutation. MPN patients showed higher blood clotting ability than the control subjects; moreover, MPN patients with the JAK2V617F mutation showed higher blood clotting ability than those without the mutation. The findings of this study indicate that the JAK2V617F mutation is correlated with the incidence of thrombosis, and analysis of this mutation has important clinical significance in the diagnosis and treatment of MPN.

Key word: Myeloproliferative neoplasm; JAK2V617F mutation; Thrombosis

\section{INTRODUCTION}

Myeloproliferative neoplasma (MPN) is a clonal disorder of hematopoiesis, characterized by the production of mature-like cells within the blood stream. Classic BCR/ ABL-negative chronic myeloproliferative disorders (MPDs) encompass three clonal diseases that arise in pluripotent hematopoietic stem cells and share clinical, hematological, and biological features: polycythemia vera (PV) (Gómez et al., 2016; McMullin et al., 2016), essential thrombocythemia (ET), and primary myelofibrosis (PMF) (Tefferi, 2010). The clinical features of MPN patients include excessive proliferation of red blood cells and platelets with splenomegaly, bleeding, bone marrow fibrosis, leukemic transformation tendency, and thrombosis. Thromboembolism seriously affects the quality of life of patients with MPN, and is the key cause of mortality (Ziakas, 2008). Early diagnosis of thrombosis is essential to reduce the mortality of patients with MPN. In 2008, the World Health Organization (WHO) proposed that the JAK2V617F mutation could be one of the diagnostic criteria of classic BCR/ ABL PMN (Tefferi and Vardiman, 2008). In this study, we assayed the JAK2V617F mutation rate and the incidence of thrombosis in MPN patients, and explored the correlation between the JAK2V617F mutation and the incidence of thrombosis.

\section{MATERIAL AND METHODS}

\section{Patients}

Patients with MPN admitted to Hematology and Oncology Department of our hospital were selected between February 2014 and February 2015. A total of 68 patients with MPN, including 31 male and 37 female patients, were recruited to this study (age range, 41-73 years; average age, 59). The patients were classified into three types of MPD based on the diagnostic classification criterion proposed by the WHO in 2008 (Tefferi and Vardiman, 2008), as follows: 37 patients with ET (17 males and 20 females; average age, 49); 23 patients with PV (12 males and 11 females; average age, 57); and 8 with PMF (2 males and 6 females; average age, 65).

Genetics and Molecular Research 15 (3): gmr.15038423 
The control group was composed of 68 individuals, among whom 21 were patients with other blood diseases such as leukemia, anemia, or infection mononucleosis, and 47 were healthy individuals (30 males and 38 females; age range: 38-73 years; average age, 57).

Written informed consent was obtained from all participants prior to enrollment in the study according to the principles of the Declaration of Helsinki; the study design was approved by the Shandong University Ethics Committee.

\section{JAK2V617F mutation test}

Peripheral blood was collected from patients with MPN and the control individuals (2-3 $\mathrm{mL}$ each) in tubes containing EDTA anticoagulant, and stored in a $-80^{\circ} \mathrm{C}$ refrigerator. Genomic DNA was extracted and amplified by PCR, according to the protocols detailed by the manufacturer in a commercial JAK2V617F mutation detection kit (Koyee Bio-Technology Co., Ltd., Shanghai, China), using a LightCycler 480 fluorescent PCR gene amplification detector (Roche, Indianapolis, IN, USA). The amplified DNA products were separated by agarose gel electrophoresis, and examined by an ultraviolet reflection transmission analyzer. Samples positive for the mutated JAK2V617F gene were sequenced by Adicon (Beijing, China).

\section{Detection of thrombosis in patients with MPN}

Factors influencing thrombosis, including the age, gender, obesity status, and blood pressure, in MPN patients were recorded and analyzed. Patients were selected according to the following criteria: age $\geq 60$ years; systolic pressure $\geq 139 \mathrm{mmHg}$ and/or diastolic pressure $\geq 89 \mathrm{mmHg}$ without using an antihypertensive drug; or blood pressure $\leq 140 / 90 \mathrm{mmHg}$ using an antihypertensive drug. Body mass index is the standard of obesity. Thrombotic disease was recorded for 1 year after MPN diagnosis.

\section{Routine blood and coagulation testing}

Peripheral blood (3-5 mL each) was collected in EDTA anticoagulant for routine blood testing using $10 \mathrm{~mL}$ sodium citrate, an AutoVue Innova kit (Johnson \& Johnson, USA), and an Automatic Blood Coagulation Analysis Instrument SF-8000 (Succeeder, China).

\section{Statistical analysis}

Statistical evaluation was performed on the SPSS 21.0 software platform (SPSS, Inc., Chicago, IL, USA). All data are reported as means \pm standard deviation (SD) of multiple iterations. The Student paired $t$-test was used to identify differences between groups; categorical variables were compared using the chi-squared test. A P value $<0.05$ was considered statistically significant; multivariate logistic regression analysis was performed.

\section{RESULTS}

\section{Screening for the JAK2V617F mutation}

The JAK2V617F mutation was detected in both patients with MPN and the control 
subjects. The results revealed the presence of the mutation in 44 of 68 patients with MPN (64.7\%; 24 patients with ET, 17 with PV, and 3 patients with PMF). However, the JAK2V617F mutation was found only in 8 of 68 subjects in the control group (11.8\%), suggesting that the rate of JAK2V617F mutation in patients with MPN was significantly higher than that in the control group. The incidences of the JAK2V617F genetic mutation are summarized in Table 1.

Table 1. Incidence of JAK2V617F genetic mutation.

\begin{tabular}{l|c|c|c|c}
\hline \multirow{2}{*}{ Group } & \multirow{2}{*}{ Number } & \multicolumn{2}{|c|}{ JAK2V617F } & Incidence of mutation \\
\cline { 3 - 4 } & & Positive & Negative & \\
\hline MPN & 68 & 44 & 24 & $64.7 \%^{*}$ \\
\hline ET & 37 & 24 & 13 & $64.9 \%$ \\
\hline PV & 23 & 17 & 6 & $73.9 \%$ \\
\hline PMF & 8 & 3 & 5 & $37.5 \%$ \\
\hline Control & 68 & 8 & 60 & $11.8 \%$ \\
\hline
\end{tabular}

*P $<0.05 v s$ the control group. MPN: myeloproliferative neoplasm; ET: essential thrombocythemia; PV: polycythemia vera; PMF: primary myelofibrosis.

\section{Incidence of thrombosis in patients with MPN}

Twenty-six of 68 patients (38.2\%) with MPN displayed symptoms of thrombosis (including 7 MPN patients with thrombotic disease), among which 13,12, and 1 were patients with ET, PV, and PMF, respectively. The type of thrombosis in these MPN patients ranged from arterial thrombosis $(\mathrm{N}=15 ; 9$ with cerebral thrombosis, 3 with coronary thrombosis, and 3 patients with superior mesenteric artery thrombosis) and phlebothrombosis $(\mathrm{N}=9 ; 5$ patients with lower extremity deep vein thrombosis, 3 with portal vein thrombosis, and 1 with splenic vein thrombosis), to arteriovenous thrombosis $(\mathrm{N}=2$; cerebral thrombus + portal vein thrombosis and cerebral thrombosis + lower extremity deep vein thrombosis).

\section{Relationship between thrombosis and JAK2V617F in patients with MPN}

The rate of JAK2V617F mutation, age, and blood pressure status of MPN patients with thrombosis was significantly higher than that of MPN patients without thrombosis $(\mathrm{P}<0.05)$. However, no significant difference was found in the age and obesity status between patients with and without thrombosis. Thrombus rates in patients with MPN are shown in Table 2.

\begin{tabular}{|c|c|c|c|c|c|c|}
\hline \multirow[t]{2}{*}{ Group } & \multirow[t]{2}{*}{ Age ( $\geq 60$ years) } & \multicolumn{2}{|c|}{ Gender } & \multirow[t]{2}{*}{ Obesity } & \multirow[t]{2}{*}{ Blood pressure } & \multirow[t]{2}{*}{ JAK2V617F } \\
\hline & & \begin{tabular}{|l|l|} 
Male & \\
\end{tabular} & Female & & & \\
\hline Thrombus & $21^{*}$ & \multicolumn{2}{|c|}{14} & 11 & \multicolumn{2}{|c|}{$23^{*}$} \\
\hline Non-thrombus & 17 & 24 & & 25 & & \\
\hline
\end{tabular}

$* \mathrm{P}<0.05$ vs the non-thrombus group.

\section{Routine blood and coagulation testing in patients with MPN}

Compared with the PV patients without gene mutation, white blood cells (WBC) and platelets were significantly increased in the patients with mutation. Compared with the ET 
patients without gene mutation, WBC and hemoglobin were significantly increased in the patients with mutation (Table 3). Moreover, the MPN patients showed higher blood clotting ability than the control group, and the JAK2V617F mutative patients showed higher blood clotting ability than the patients without mutation among the MPN patients (Table 4).

Table 3. Blood results from patients with myeloproliferative neoplasm.

\begin{tabular}{|c|c|c|c|}
\hline Group & WBC $\left(10^{9} / \mathrm{L}\right)$ & PLT $\left(10^{9} / \mathrm{L}\right)$ & HGB $(\mathrm{g} / \mathrm{L})$ \\
\hline \multicolumn{4}{|c|}{ JAK2V617F positive } \\
\hline ET & $32.89 \pm 4.25^{*}$ & $881.41 \pm 320.23$ & $161.92 \pm 16.19^{*}$ \\
\hline $\mathrm{PV}$ & $21.98 \pm 1.95^{*}$ & $364.68 \pm 97.72 *$ & $178.33 \pm 17.21$ \\
\hline PMF & $14.36 \pm 3.01$ & $179.85 \pm 33.56$ & $142.12 \pm 4.19$ \\
\hline \multicolumn{4}{|c|}{ JAK2V617F negative } \\
\hline ET & $16.11 \pm 2.48$ & $693.52 \pm 237.29$ & $144.13 \pm 20.62$ \\
\hline $\mathrm{PV}$ & $13.98 \pm 1.34$ & $216.39 \pm 89.74$ & $137.96 \pm 13.21$ \\
\hline PMF & $13.25 \pm 1.17$ & $156.74 \pm 18.32$ & $128.93 \pm 1.37$ \\
\hline
\end{tabular}

$* \mathrm{P}<0.05 v s$ the JAKV617F negative group. ET: essential thrombocythemia; PV: polycythemia vera; PMF: primary myelofibrosis; WBC: white blood cell; PLT: platelet; HGB: hemoglobin.

Table 4. Results of the routine coagulation test of patients with myeloproliferative neoplasm (MPN).

\begin{tabular}{l|c|c|c|c}
\hline Group & PT & APTT & TT & FIB $(\mathrm{g} / \mathrm{L})$ \\
\hline MPN patient & $16.64 \pm 3.98^{*}$ & $58.91 \pm 21.19^{*}$ & $21.01 \pm 3.53^{*}$ & $2.81 \pm 1.22$ \\
\hline JAK2V617F positive & $16.13 \pm 3.41^{\#}$ & $62.29 \pm 23.55^{\#}$ & $20.43 \pm 4.12^{\#}$ & $2.19 \pm 1.52$ \\
\hline JAK2V617F negative & $14.97 \pm 2.02$ & $49.83 \pm 8.36$ & $18.36 \pm 2.13$ & $2.18 \pm 0.71$ \\
\hline Control & $13.98 \pm 0.89$ & $33.69 \pm 5.71$ & $17.09 \pm 2.32$ & $2.41 \pm 0.82$ \\
\hline
\end{tabular}

${ }^{*} \mathrm{P}<0.05$ vs the control group; ${ }^{\mathrm{P}}<0.05$ vs JAK2V617F negative group. PT: prothrombin time; APTT: activated partial thromboplastin time; TT: thrombin time; FIB: fibrinogen.

\section{DISCUSSION}

MPN is a clonal hematopoietic stem cell disease characterized by sustained proliferation of bone marrow cells (Tefferi et al., 2007). In this disease, blood cells proliferate significantly because of the excessive proliferation of, and apoptosis inhibition in, bone marrow hematopoietic stem cells. Blood cell proliferation causes an increase in hematocrit, blood viscosity, and platelet count, leading to serious complications such as bleeding and thrombus embolism. Carobbio et al. (2007) reported that leukocytes promote thrombosis. Neutrophils and platelets form complexes to regulate the expression of platelets, such as P-selectin and tissue factor, thereby promoting the activation of vascular endothelial cells. A previous study suggested that mortality caused by thrombosis accounted for $35-70 \%$ of the total mortality in MPN patients (Park et al., 2013). Therefore, it is a condition that generates significant complications for MPN patients. Our results showed that 26/68 (38.2\%) MPN patients, including 7 with thrombotic disease, showed thrombosis.

Finazzi et al. (2007) reported that the incidence of thrombus in patients with the JAK2V617F mutation is higher than that in patients without this mutation. Kralovics et al. (2005) also reported that patients expressing the JAK2V617F mutation are more susceptible to complications such as thrombosis. Therefore, the JAK2V617F mutation is the most remarkable genetic mutation in MPN. The JAK2V617F mutation was first identified in patients with MPN in 2005 (Reikvam and Tiu, 2012; Bogani et al., 2013), and 
was proposed as a diagnostic criterion for BCR/ABL-negative MPN by the WHO in 2008 . However, the pathogenesis of the JAK2V617F mutation in classic BCR/ABL-negative MPN remains unclear. The current opinion leans towards the JAK2V617F mutation theory. The high frequency point mutations in JAK2 exon 12 ( $\mathrm{G}$ mutates into $\mathrm{T}$ at site 1849) are believed to lead to a valine changing into a phenylalanine at site 617 of the JAK2 kinase domain structure. This change causes instability in, and abnormal activation of, the JH2 structure and further activation of transcription in the cell proliferation gene via activation of the JAK/STAT signal transduction pathways, resulting in sustained cellular proliferation (Harrison et al., 2012; Evrot et al., 2013; Hsiao et al., 2013). In this study, we showed that the mutation was found in 44 of 68 patients with MPN (64.7\%), including $24 \mathrm{ET}, 17 \mathrm{PV}$, and $3 \mathrm{PMF}$ patients. These results indicate the high incidence rate of the JAK2V617F mutation in patients with MPN.

In this study, we evaluated several factors affecting the incidence of thrombosis in patients with MPN, such as the age, gender, obesity status, blood pressure, and the JAK2V617F mutation. Our investigation revealed that patients with thrombosis showed significantly higher rates of the JAK2V617F mutation, were of a greater age, and had higher blood pressure than patients without thrombosis had. These results suggest that the JAK2V617F mutation is an important risk factor for the incidence of thrombosis in MPN patients. The results of the routine blood test showed a significant increase in WBCs in patients with PV and ET expressing the mutation, indicating that WBCs may play an important role in the incidence of thrombosis in MPN patients. The results of the routine coagulation test revealed that MPN patients with the JAK2V617F showed a significantly higher blood clotting ability than those without the mutation. This result suggests that patients with MPN show abnormal coagulant function, which should be investigated in future studies.

There are a few limitations to this study. First, the study population was relatively small, which may have limited the statistical power for the identification of differences between groups. Second, although we have demonstrated the correlation between the JAK2V617F mutation and the incidence of thrombosis, we did not clarify the underlying mechanism, which is worthy of further study. Third, the results of this observational study should be confirmed by larger clinical trials in multiple clinical centers.

In conclusion, JAK2V617F is an important diagnostic standard in patients with MPN; therefore, the detection of this mutation has important clinical significance. JAK2V617F participates in the development of MPN, and is correlated with thrombotic disease in MPN patients. Therefore, testing for the JAK2V617F mutation could help identify the incidence of thrombotic disease in MPN patients, and could be helpful in the clinical diagnosis and treatment of MPN.

\section{Conflicts of interest}

The authors declare no conflict of interest.

\section{ACKNOWLEDGMENTS}

We would like to thank the patients for their willing participation, and the laboratory technicians for their valuable efforts.

Genetics and Molecular Research 15 (3): gmr.15038423 


\section{REFERENCES}

Bogani C, Bartalucci N, Martinelli S, Tozzi L, et al.; Associazione Italiana per la Ricerca sul Cancro AGIMM Gruppo Italiano Malattie Mieloproliferative (2013). mTOR inhibitors alone and in combination with JAK2 inhibitors effectively inhibit cells of myeloproliferative neoplasms. PLoS One 8: e54826. http://dx.doi.org/10.1371/journal. pone. 0054826

Carobbio A, Finazzi G, Guerini V, Spinelli O, et al. (2007). Leukocytosis is a risk factor for thrombosis in essential thrombocythemia: interaction with treatment, standard risk factors, and Jak2 mutation status. Blood 109: 2310-2313. http://dx.doi.org/10.1182/blood-2006-09-046342

Evrot E, Ebel N, Romanet V, Roelli C, et al. (2013). JAK1/2 and Pan-deacetylase inhibitor combination therapy yields improved efficacy in preclinical mouse models of JAK2V617F-driven disease. Clin. Cancer Res. 19: 6230-6241. http://dx.doi.org/10.1158/1078-0432.CCR-13-0905

Finazzi G, Rambaldi A, Guerini V, Carobbo A, et al. (2007). Risk of thrombosis in patients with essential thrombocythemia and polycythemia vera according to JAK2 V617F mutation status. Haematologica 92: 135-136. http://dx.doi. org/10.3324/haematol.10634

Gómez M, Guillem V, Pereira A, Ferrer-Marín F, et al. (2016). Risk factors for non-melanoma skin cancer in patients with essential thrombocythemia and polycythemia vera. Eur. J. Haematol. 96: 285-290. http://dx.doi.org/10.1111/ ejh. 12588

Harrison C, Kiladjian JJ, Al-Ali HK, Gisslinger H, et al. (2012). JAK inhibition with ruxolitinib versus best available therapy for myelofibrosis. N. Engl. J. Med. 366: 787-798. http://dx.doi.org/10.1056/NEJMoa1110556

Hsiao HH, Liu YC, Yang MY, Tsai YF, et al. (2013). Decreased expression of PIAS1 and PIAS3 in essential thrombocythemia patients. Genet. Mol. Res. 12: 5617-5622. http://dx.doi.org/10.4238/2013.November.18.10

Kralovics R, Passamonti F, Buser AS, Teo SS, et al. (2005). A gain-of-function mutation of JAK2 in myeloproliferative disorders. N. Engl. J. Med. 352: 1779-1790. http://dx.doi.org/10.1056/NEJMoa051113

McMullin MF, Wilkins BS and Harrison CN (2016). Management of polycythaemia vera: a critical review of current data. Br. J. Haematol. 172: 337-349. http://dx.doi.org/10.1111/bjh.13812

Park SH, Chi HS, Cho YU, Jang S, et al. (2013). The allele burden of JAK2 V617F can aid in differential diagnosis of Philadelphia Chromosome-Negative Myeloproliferative Neoplasm. Blood Res. 48: 128-132. http://dx.doi. org/10.5045/br.2013.48.2.128

Reikvam H and Tiu RV (2012). Venous thromboembolism in patients with essential thrombocythemia and polycythemia vera. Leukemia 26: 563-571. http://dx.doi.org/10.1038/leu.2011.314

Tefferi A (2010). Novel mutations and their functional and clinical relevance in myeloproliferative neoplasms: JAK2, MPL, TET2, ASXL1, CBL, IDH and IKZF1. Leukemia 24: 1128-1138. http://dx.doi.org/10.1038/leu.2010.69

Tefferi A and Vardiman JW (2008). Classification and diagnosis of myeloproliferative neoplasms: the 2008 World Health Organization criteria and point-of-care diagnostic algorithms. Leukemia 22: 14-22. http://dx.doi.org/10.1038/ sj.leu.2404955

Tefferi A, Thiele J, Orazi A, Kvasnicka HM, et al. (2007). Proposals and rationale for revision of the World Health Organization diagnostic criteria for polycythemia vera, essential thrombocythemia, and primary myelofibrosis: recommendations from an ad hoc international expert panel. Blood 110: 1092-1097. http://dx.doi.org/10.1182/ blood-2007-04-083501

Ziakas PD (2008). Effect of JAK2 V617F on thrombotic risk in patients with essential thrombocythemia: measuring the uncertain. Haematologica 93: 1412-1414. http://dx.doi.org/10.3324/haematol.12970

Genetics and Molecular Research 15 (3): gmr.15038423 